\title{
Bladder Stone On Non-Absorbable Sutures Twenty Years After A Female Sterilization.
}

\section{Thirukumar $M^{1}$}

${ }^{1}$ Faculty of Health Care Science, Eastern University

\begin{abstract}
;
Foreign body in the urinary bladder causes recurrent infection and stone formation. This is an unusual case report of a bladder stone formed on a non-absorbable suture 20 years after a female sterilization. It was detected with ultrasound and pelvic X-Ray. Laparotomy was done for its completed removal as cystoscopy failed to remove it. This case highlights that abdominal- pelvic scan and pelvic x-ray in addition to a urine test, should be included in the early part of the investigation in the management of recurrent urinary tract infection to exclude the possibility of the bladder stone
\end{abstract}

\section{Key word}

urinary bladder stone, urinary tract infection, female sterilization, foreign body.

\section{Introduction}

The urinary bladder stones are uncommon among females. It usually occurs due to bladder outlet obstruction and rarely from a foreign body. There can be various types of foreign bodies. These foreign bodies in the urinary bladder cause recurrent urinary tract infection and cause challenge in diagnosis and management. Foreign bodies may enter the bladder by ascending from the urethra, descending from the ureter or migration from adjacent organs $(1,2)$.

Several foreign bodies in the bladder have been reported in the literature (3). They are inserted under various instances such as children self- insert for inquisitiveness and woman with disinhibited behavior insert for eroticism. It can also be a consequence of disturbed behaviour such as in psychiatric illness, senile states and under the influence of drugs particularly, alcohol. This is a case report of a bladder calculus twenty years after a female permanent sterilization.

\section{Case}

A 56-year-old woman presented with recurrent urinary tract infection for 2 years duration. She had six culture positive urinary tract infection episodes during the previous period of two years. Therefore, she was referred to the gynaecology outpatient clinic to exclude possibility of genital prolapse as a cause of recurrent urinary tract infections.

She had previous four vaginal birth and the age of last child is 20 years. Further, she underwent permanent sterilization (Bilateral tubal resection and ligation) following her last childbirth. Other than this, she never underwent any surgeries in the past. Her general clinical examination, cardiovascular, respiratory and abdominal examinations were unremarkable. During vaginal examination, a Nylon thread was noted through the urethra.

Ultrasonography had not detected any renal tract abnormalities. However, there was an echogenic object in the bladder; a suspected foreign body within the urinary bladder (Figure 1). The pelvic $\mathrm{x}$ ray showed a calcified mass within the urinary bladder as a faint radio-opaque shadow in the bladder area. (figure 2). Then a cystoscopy was performed to look for any foreign bodies (Figure 3) and to remove the objects from within the bladder. An encrusted bladder stone on non-absorbable sutures was noted hanging from the anterior surface of the urinary bladder near its dome. This bladder stone with its suture is fixed on the bladder wall and difficult to remove.

The object was incompletely removed with cystoscopic grasping forceps. An open removal via suprapubic cystotomy was required for its complete removal. Her post-operative period was unremarkable and repeat urine culture three weeks following the surgery did not show any organism 
Figure 1

Ultrasonography of the urinary bladder;

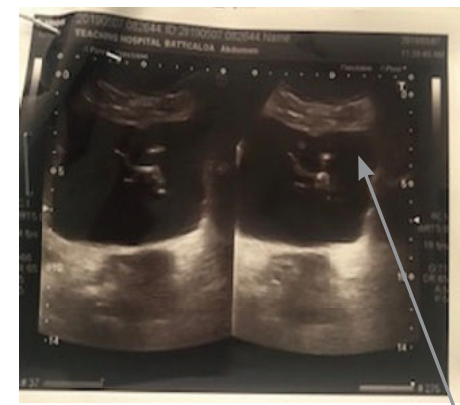

an echogenic object in the bladder

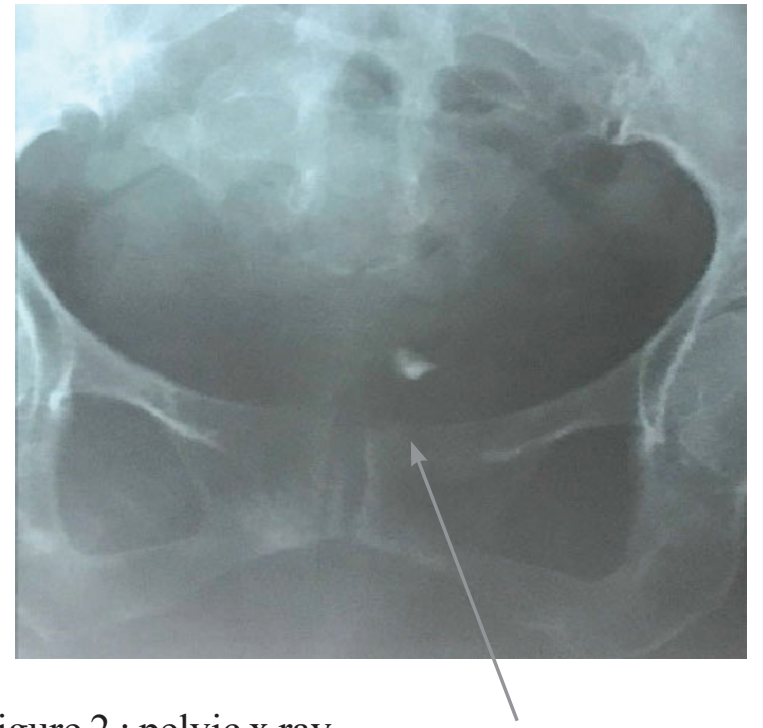

Figure 2 : pelvic $x$ ray calcified mass within the urinary bladder

Figure 3 cystoscopy;

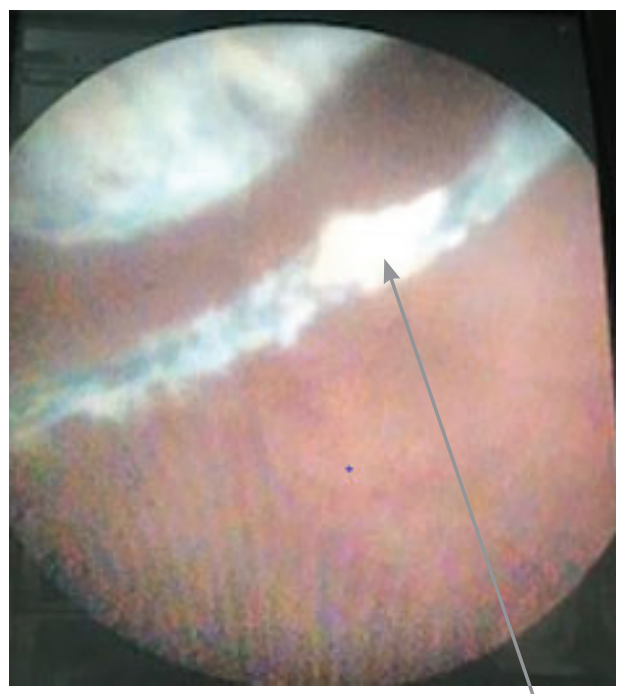

Encrusted bladder stone on non-absorbable sutures hanging from the anterior surface of the urinary bladder near its dome.
Figure 4 Removed stone with part of suture material

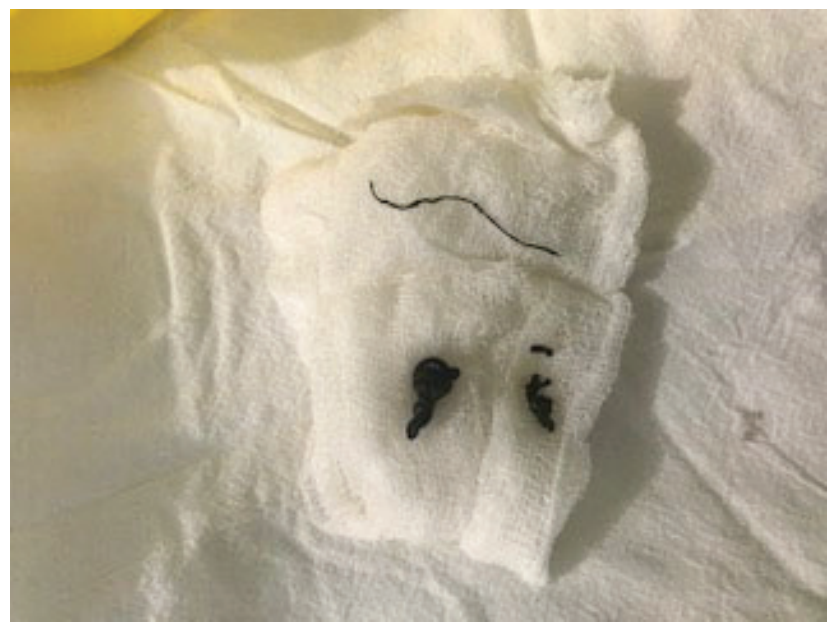

\section{Discussion}

A long -standing foreign bodies such as suture material in the urinary bladder predisposes to recurrent urinary infections and encrustations with stone formation, as it happened in present case.

Further, foreign bodies in the urinary bladder can present acute symptoms such as dysuria, haematuria, frequency and urethral discharge. Sometimes they have delayed presentation such as calcified stitch after Stamey procedure, intrauterine contraceptive device, suprapubic catheter tip sheared off, by trochar. These clinical presentations can be delayed between 6 months to 5 years after the introduction of the foreign body (4).This reported case presented 20 years after the surgery and so far no case was reported after such a long interval.

Intravesical foreign bodies should be considered in the differential diagnosis of recurrent lower urinary tract infection. These foreign bodies can be self-introduced, result of medical errors, result of a penetrating injury or even it could have been migrated from the surrounding organs. Almost any foreign body placed in the vicinity of the bladder has a potential to migrate into the urinary bladder such as intrauterine contraceptive device, vaginal pessary, artificial urinary sphincter, prosthetic slings, nonabsorbable sutures used in Stamey procedures and surgical gauze. (5-8). Stones are formed when these sutures are exposed in the bladder cavity. However, monofilament polypropylene sutures do not cause such reactions (9). The suture was seen with completely encrusted in stone. There could be two possibilities causing this clinical situation. One possibility is the direct 
injury to the bladder incurred during the surgery with nonabsorbable sutures and became a nidus. Second possibility is that the suture immediately outside the bladder could have entered the bladder over a period of several years by eroding the bladder wall and formed a nidus for stone formation and recurrent urinary tract infection. Since this patient presented with symptoms almost 20 years after the surgery the second possible mechanism could explain its pathogenesis.

The objects protruding the urethra should be pushed into the bladder and examined cystoscopically prior to removal (10). This case was also similarly managed. The cystoscopy management is the main treatment for a high success rate. It is also an effective and safe method for removal of bladder stones. The main principle for treating a bladder stone is to remove the underlying cause of stone formation. Therefore, management is aimed for complete extraction of the foreign body with minimal trauma to the bladder and urethra. However, a bladder stone resulting from a foreign body that has become fixed on the bladder wall may require a laparotomy for its removal. The reported case also managed with cystoscopy followed by laparotomy for its complete removal of the foreign body.

According to the literature, bladder stones caused by foreign bodies involving sutures contain components such as calcium, ammonium, phosphate, oxalate, and uric acid. These components mostly exist as mixtures. Unfortunately, the stone of this case had not been sent biochemical analysis due to the limited resource in our hospital.

\section{Conclusions}

This case highlights that high index of suspicion on foreign body and bladder stones should be there in managing the recurrent urinary tract infections in females,

especially those underwent previous abdominopelvic surgeries. Further, in addition to a urine test, abdomino pelvic scan and pelvic $\mathrm{x}$-ray should be included in early part of the investigations to exclude the possibility of the bladder stone in the management of recurrent urinary tract infection. It avoids the delay in diagnosing the stone with or without its aetiology such as foreign bodies.

\section{References}

1. Sheng-Tsun Su1, He-Fu Huang1, Shu-Fen Chang2. Encrusted Bladder Stone on Nonabsorbable Sutures after a Cesarean Section: A Case Report; JTUA 2009_20_No. 3 NQP.

2. Dilip K. Pal, Asim K. Bag, Intravesical wire as foreign body in urinary bladder, Int Braz J Urol. $2005 ; 31: 472-4$

3. Eckford SD, Persad RA, Brewster SF, Gingell JC: Intravesical foreign bodies: five- year review. Br J Urol. 1992; 69:41-45.

4. S. D. ECKFORD, R. A. PERSAD, S. F. BREWSTER and J. C. GINGELL. Intravesical Foreign Bodies: Five-year Review.British Journolof Urology (1992), 69,4145

5. Bartoletti R, Gacci M, Travaglini F, Sarti E, Selli C. Intravesical migration of AMS 800 artificial urinary sphincter and stone formation in a patient who underwent radical prostatectomy. Urol Int. 2000; 64:167-8.

6. Tornero J, Palou J, Prados M, Salvador J, Vicente J.Bladder perforation caused by foreign body migration. Int Urol Nephrol. $2000 ; 32: 241-3$.

7. Nouira Y, Rakrouki S, Gargouri M, Fitouri Z,Horchani A. Intravesical migration of an intrauterine contraceptive device complicated by bladder stone a report of six cases. Int Urogynecol J Pelvic Floor Dysfunct. 2007; 18:575-8.

8. Chamary VL. An unusual cause of iatrogenic bladder stone. Br J Urol. 1995; 76:138.

9. Morris MC, Baquero A, Redovan E, et al. Urolithiasis on absorbable and non-absorbable suture materials in the rabbit bladder. J Urol $1986 ; 135: 602-3$.

10. Kochakarn W1, Pummanagura W, Foreign bodies in the female urinary bladder: 20-year experience in Ramathibodi Hospital. Asian J Surg. 2008 Jul;31(3):130-3. 